\title{
Spatial-temporal Indexing Research based on Road Network: Improved-MON-tree
}

\author{
Ma Chi ${ }^{1,2, *}$, Huang Yubin ${ }^{3}$, Zhu Yongyong ${ }^{4}$ and Yu Yanhui ${ }^{1}$ \\ ${ }^{1}$ College of Software, University of Science and Technology LiaoNing, Anshan, China \\ ${ }^{2}$ Dongling School of Economics and Management, University of Science and \\ Technology Beijing, Beijing, China \\ ${ }^{3}$ Anshan Iron and Steel Group Corporation, Anshan, China \\ ${ }^{4}$ Department of Economics and Business Administration, Chongqing University of \\ Education, Chongqing, China \\ asmachi@126.com

\section{Abstract}

Contrary to MON-tree index less than the temporat and spatial indexing methods based on road networks we proposed Improved-MON-tree. The index is divided into two levels and the quad tree grid index at the top of the road network, and the bottom of the two-dimensional $R$ tree index moving object moving information. In order to support the global trajectory of moving objects query, add a hash structure underlying index structure connected to the moving object information. Verified bycexperiment uder certain conditions, Improved$M O N$-tree than the MON-tree based on edge and routes, has good spatial-temporal window query performance and global trajectory.

Keywords: Moving Objects; Spatial-Temporal Index; Network Model; Improved-MONtree

\section{Introduction}

Moving object database is a branch of the spatial-temporal database research that has been developed recent years, it is also the technical basis of the location-related services, established efficient moving object index is one of the key technologies of the moving object database [1]. The spatiat-temporal database is a spatial database [2, 3] extension in the time dimension. In recent years, people come to realize the importance of network constrained moving objects, and proposed a number of moving object database index based on the road network. The proposed index structure can be roughly divided into four categories:the first is for global trajectory index moving objects in the network [4-6].; the second is the index for future trajectory [7]; the third index for historical trajectory of moving objects in the network [8-14]: the fourth is in real-time location information [15].

MON-tree index structure using hierarchical idea, the upper road network using a 2dimensional R-tree index, the lower moving objects using 2-dimensional R-tree forest index moving information. And given two road modeling methods: one is based on edge of the index structure, one is based on the route structure. Excessive based on the index structure of the edge of the lower number of R-tree forests, the trajectory of moving objects query costs too much; too much dead space on route-based. The Improved-MON-tree index combines

\footnotetext{
*Corresponding Author: asmachi@126.com
} 
advantages based on the edge-based and routed. The upper index structure using quad tree of road network space, the lower uses 2-dimensional R-tree forest index moving objects and the underlying index structure using a hash structure to organizations moving object trajectory information for global trajectory of the object query.

This article is organized as: Section 2 is the road network modeling. Section 3 we propose the Improved-MON-tree. Section 4 experimentally evaluates our proposed index structure and compares them to the corresponding MON-tree. Finally, Section 5 concludes the article.

\section{Network Modeling}

Temporal-spatial data model is the basis to create a space-time index, spatial data model for the traffic and road network, in this article, we will be divided into the road network model is used to reflect the spatial data and reflects the trajectory of moving objects spatiotemporal data model.

\subsection{Network Road Model}

The most widely used road network model in two ways: based on the route of the road network model based on edge of the road network model. Analysis under the first section of the advantages and disadvantages of the two road hetwork model, the mixed road network model is used. The relevant definitions are as follows.

Definition1: Let $G=(R, J)$ represent an urban road network, where $R$ is a collection of the curve of the road, $J$ is the intersection set 41

Definition2: Let route $=($ rid, $r p t r, 1, n, y, E)$ is a route [4], where rid uniquely identifies the route. rptr on behalf of the current route of the geometric shape descriptors composed by planar point set $X \times Y$. len represen s the length of the current route. $J$ is the collection of all current route intersection as well as the current position of intersection in the route, the specific form ( $j i d$, pos), jid representatives uniquely identifies the intersection, pos is the relative position of the current intersection curve in the road above, do normalized 1 treatment, that is, the length of all roads 1, pos within a real number. $E$ represents the current set of road sections aboye.

Definition 3: Let ed $g$ e $=\left(\right.$ edgeid, kind $,\left(j_{s}\right.$, pos $\left._{s}\right),\left(j_{e}\right.$, pos $\left.\left._{e}\right)\right)$ represent an edge polyline, where edgeid represents the unque identifier of the current edge; kind representative of the current edge is unidirectional or bidirectional; $\left(j_{s}, p o s_{s}\right)$ and $\left(j_{e}, p o s_{e}\right)$ respectively represent the start and end of the current edge intersection.

Definition4: Let $j=\left(j\right.$ id, location, $\left.\left(\left(\text { rid }_{j}, \text { pos }_{j}\right)\right)_{j=1}^{n}, m x\right)$ is an intersection[4], where jid represents the unique identifier of the intersection; location represents the current intersection position- coordinates; $\left(\left(\text { rid }_{j}, \text { pos }_{j}\right)\right)_{j=1}^{n}$ expressed after all routes in the intersection curve and the intersection of the relative position in the route; $m x$ is the intersection of the adjacency matrix [16], to describe the connectivity and the traffic rules of the current route intersection curve.

Definition5: Let g pos $=(r i d, d)$ is a position in the network[11] in the road network, the rid is road sign. $d \in[0,1]$ represents the road position, the actual position is len $\times d$, len is the length of the route. 


\subsection{Temporal-spatial Data Model}

Amount of data in order to reduce the time and space of the moving object, using temporal data model similar to the Mon-tree to index moving objects to the route trajectory fragments, so that, the time and space are not stored in the database of a discrete movement point, but to track fragments as the basic unit of storage.

Definition 6: Let $m v_{i k}=\left(\right.$ rid $\left._{\text {, } t i m e_{s}}, t i m e_{e}, p o s_{s}, p o s_{e}, \bar{v}\right)$ is the $k t$ h running trajectory of the moving object $\mathrm{i}$, where rid for the current route identification. time $e_{s}$ and time $e_{e}$ show the moving object time of into route and leave the current route, pos and pos $_{e}$ are current relative position of the route of the current moving object, and its value is a real number between 0 to $1 ; \bar{v}$ is this trajectory of movement speed vector.

\section{Improved-MON-tree Index Structure}

Improved-MON-tree index structure is divided into two layers. The upper layer is constituted by a quadtree and hash hash structure, index the road network. Zoning policies upper the quadtree index of road network, first to the entice network is divided into four regions, then the number of judgments sections according to each region of the threshold $\Phi$, if more than $\Phi$ continue to divide, until the entire road network is divided into each leaf node is reached.Top quadtree leaf node tuple form 〈mb polyline bounding rectangle of the edge, polylinePtr foint to the road polyline storage address, ptr is the pointer to point to the underlying Reree, Internal nodes have form $<\mathrm{mbr}, \operatorname{child} \mathrm{Ptr}>$, where mbr is minimum bounding rectangle of the current node contains children node, childPtr is the pointer to point to the ehildren node. Hash structure tuple information, where edgeID is the polyline identification and treePtcis a pointer to the top quadtree leaf node and used to marking whether polyline is constracted. The hash structure is organized by edgeID. The top hash structure is different from MON-tree. Then, the top quadtree leaf nodes index edge-polyline, many nodes (top quadtree) is point to one R-tree (bottom R-tree), because a route is consisted by many edges.

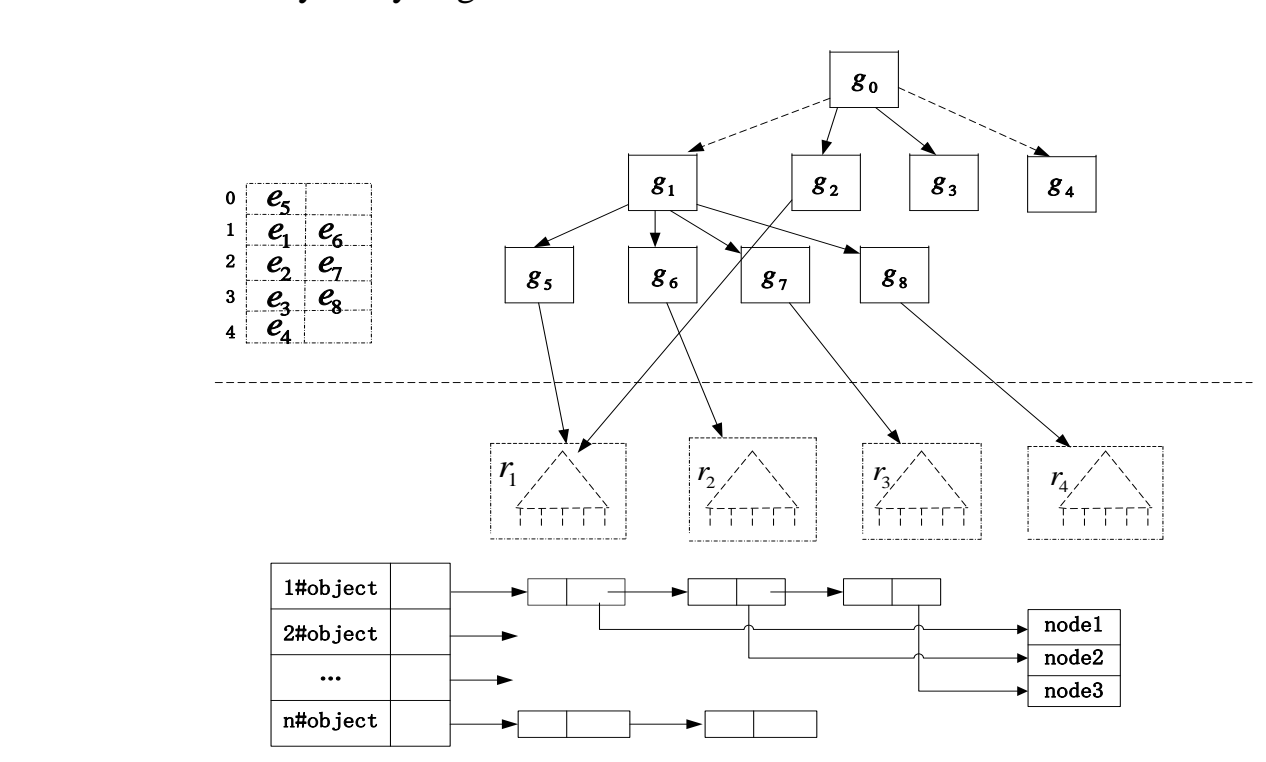

Figure 1. Improved-MON-tree Structure 
Bottom 2DR-tree index objects' movements along a route. Its leaf nodes have the form $<$ mid, mbr, polylinePtr, edgeID, rID, mvptr > where mid is the unique identification of the moving objects, mbr represents the movements of objects, it is composed by a time interval $\left(t_{\text {enter }}, t_{\text {end }}\right)$ and the position interval ( pos $_{\text {enter }}$, pos $\left.s_{\text {end }}\right)$. edgeID and rID are respectively the edge and route identification. mvptr is a pointer to point the current object running trajectory vector. Therefore, multiple top leaf nodes are corresponding to bottom 2DR-tree. The hash + single linked list structure to record the running trajectory vector of each moving object. The hash has tuple form $<$ mID, nodePtr $>$, where mID is the moving object identification and nodePtr is the head node of a linked list which storage this object' movement trajectory. The bottom of the hash is used to meet the query of an object's global trajectory. The structure is showed in Figure 1.

\subsection{Set up Index}

3.1.1. Polyline Insert: Construction of the road network is the edge of the insertion process. First, according to identification of object insert into the hash structure and its pointer set empty. The insertion of the edge in the top quadtree is postponed to first moving object traversing it. The reasons for doing so: to avoid the top quadtree don't have moving objects.

3.1.1. Polyline Insert: The moving object insertion proces 3 divided into bottom R-tree insertion and build process linked list with each object trajeetory. First, according to the moving objects' MBR inserted into the corresponding position of the bottom R-tree. Then, based on the identification attribute of a road edge of the eurrent object to check the top hash structure to find associated edge, if it is empty, then the current edge inserted top quadtree, and updates the hash structure to pointer to thistop quadtree node else perform the next step. Finally, in the bottom hash structure to retrieve the current moving object identification, if not registered object identification in the hash structure and then assign it to a linked list, and the basic information of moying objects link to the current linked list. If the hash structures stored in the identity of the current moving object, then insert the information about the current moving object into the current list.Moving objects insertion algorithm is as follows:

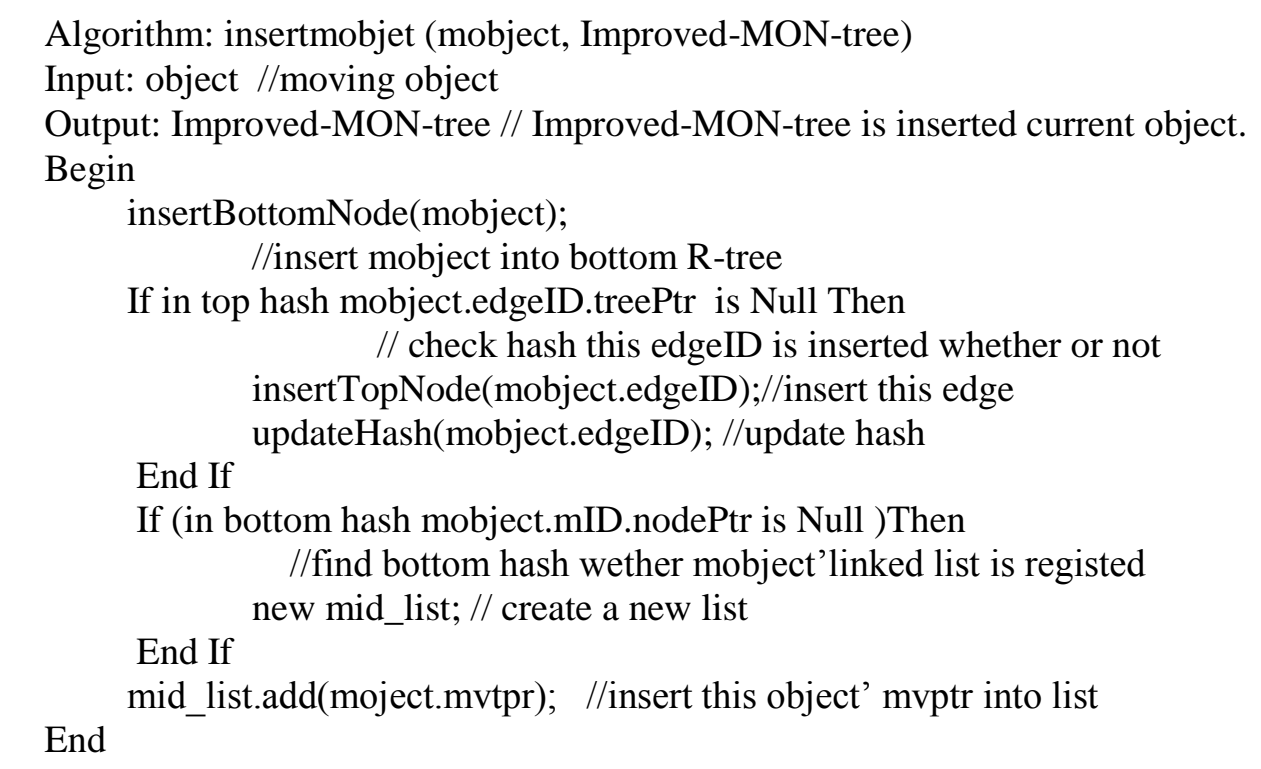




\subsection{Query}

In this paper we give the process of region query, as well as the global trajectory query about specific moving object. Here we describe each query.

3.2.1. Window Query: For spatiotemporal query query, set the query input query $=\left(x_{1}, x_{2}, y_{1}, y_{2}, t_{1}, t_{2}\right)$. Query steps:1. With window $=\left(x_{1}, x_{2}, y_{1}, y_{2}\right)$ query Improved-MON-tree top quadtree, find corresponding edge of polylinePtr pointer specific road information has been calculated to get a set sequence $\left(\text { rid }{ }_{j} \times\left(p_{j}, p_{j+1}\right)\right)_{j=1}^{n} ; 2$.For the first step sequence add time intervals to get $\left(\mathrm{rid}_{i}, p_{i}, p_{i+1}, t_{1}, t_{2}\right)^{n}{ }_{j=1}$.In corresponding bottom R-tree query which mbr is intersected with $\left(\operatorname{rid}_{i}, p_{i}, p_{i+1}, t_{1}, t_{2}\right)_{j=1}^{n}$, and output the corresponding moving object identification. Algorithm is as follows.

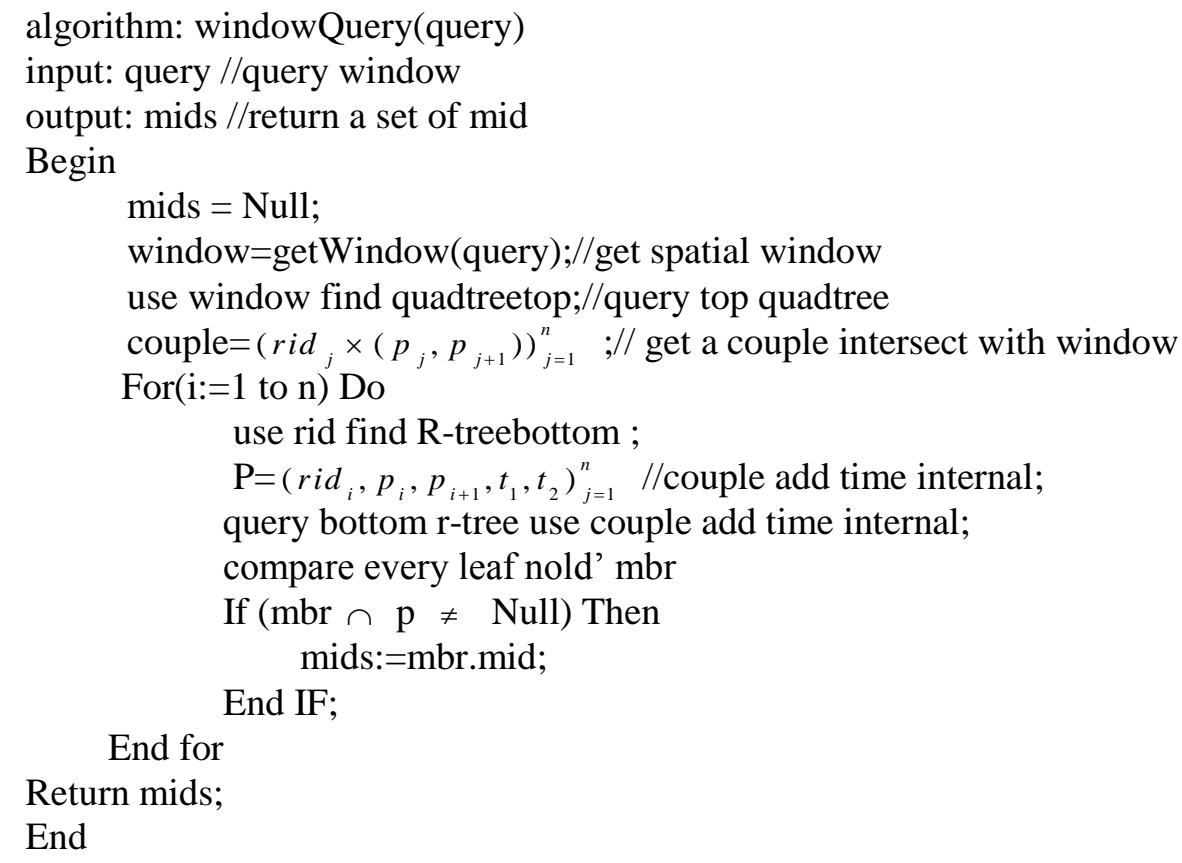

3.2.2. Window Query: For a given global trajectory query of the object: query 7 gettrajectory (mid). Its meaning is query given the running track of the moving objects. Query for the global trajectory information given object, do not traverse the entire bottom R-tree according to the mid to bottom the hash + single linked list structure traversal, get the head of the list node, scratch traversal to last all information obtained. Steps: 1.get single linked list head node, depending on the bottom hash function. 2 start traversing from the list head node to the end node. Algorithm is as follows.

algorithm :getTrajectory(mid)

input: mid //object identification

output: object.moveInfo //return trajectory information

Begin

object.moveInfo=null; 
If (mid in bottom hash is Null )Then;

//bottom hash don't have this mid

break;

return object.moveInfo=null ;

End if

node=list head node; //get list head node

while(node.next != null) Do // traverse list

object.moveInfo:=object' trajectory info; //get trajectory

End while

Return object.moveInfo;

End

\section{Experimental Analysis}

In order to understand the performance of the Improved-MON-tree, we compared Improved -MON-tree with edge-oriented and route- oriented MON-tree.Test environment: win7 64-bit operating system, Iterl(R) Core(TM) i5-3360M CPU @2.80GJiz, 4GB RAM. Experimental platform for database developed by the University of Hagen, Germany SECONDO [17]. Index is implemented by $\mathrm{C}++$ program.

Table 1. Parameter of the Experiment

\begin{tabular}{cc}
\hline Parameter & Value \\
\hline Number of moving objects & 1000200040006000 \\
Number of routes & 1784 \\
Number of edges & 3874 \\
Window query size & $5 \% \quad 10 \% \quad 15 \% \quad 20 \%$ \\
\hline
\end{tabular}

Experiments mainly compare index size, the window query efficiency and trajectory query performance. Experiments use the parameters shown in Table 1. Experiments generate respectively the number of moying bject is 1000 to 6000 trajectory within $4 \mathrm{~h}$, these trajectories are unifornly distributed throughout the road network. The route number of 1784 , edge number 3874

Due to in the bottom Improved-MON-tree adds hash + single list structure, ImprovedMON-tree index structare algorithm compared to the edge-oriented and road-oriented MONtree is slightly morecomplex, so Improved-MON-tree in the index size compared to MONtree needs to be optimized. The relationships of index size between the numbers of moving objects as show in Figure 2.

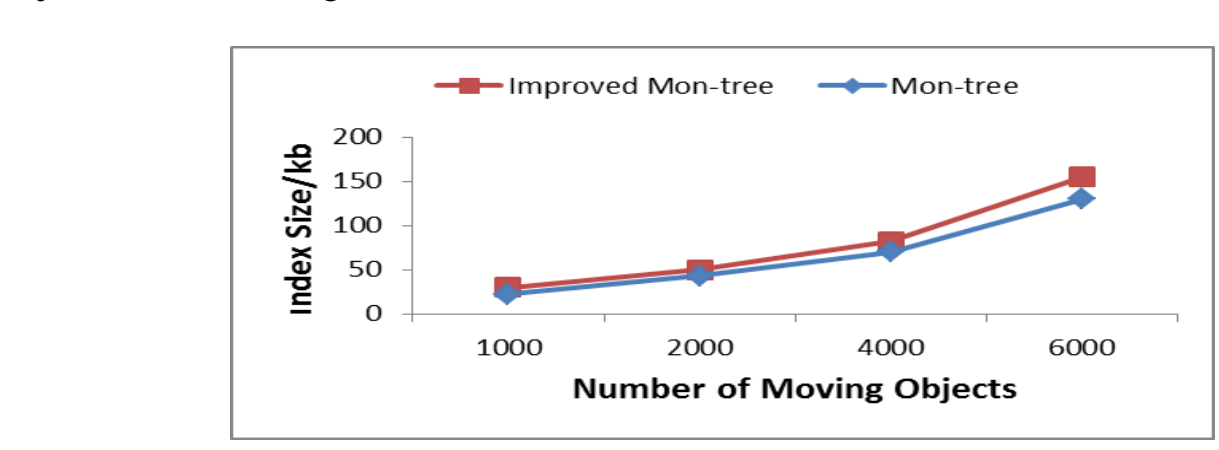

Figure 2. Index Size vs Numbers of Objects 
For the query window, we select the entire $15 \%$ of the road network as a query region, the time zone for the entire time range of 5\%. The numbers of moving objects from 1000-6000 distribute, and the response time to change trend are as following, with the change in the number of moving objects shown in Figure 3.

Window query we also looked at the number of moving objects fixed window area ranging from $5 \%$ to $20 \%$ and the time zone for the entire time range of 5\%. The number of moving objects is fixed at 4000, the response time and the relationship between the size of the window are shown in Figure 4.

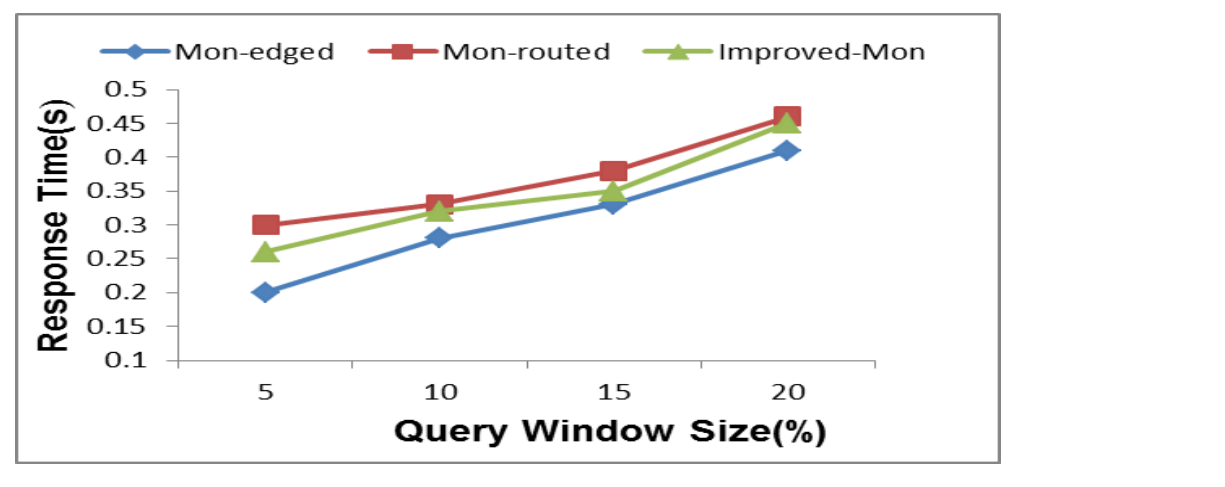

Figure 3. Response Time Numbers of Mojects

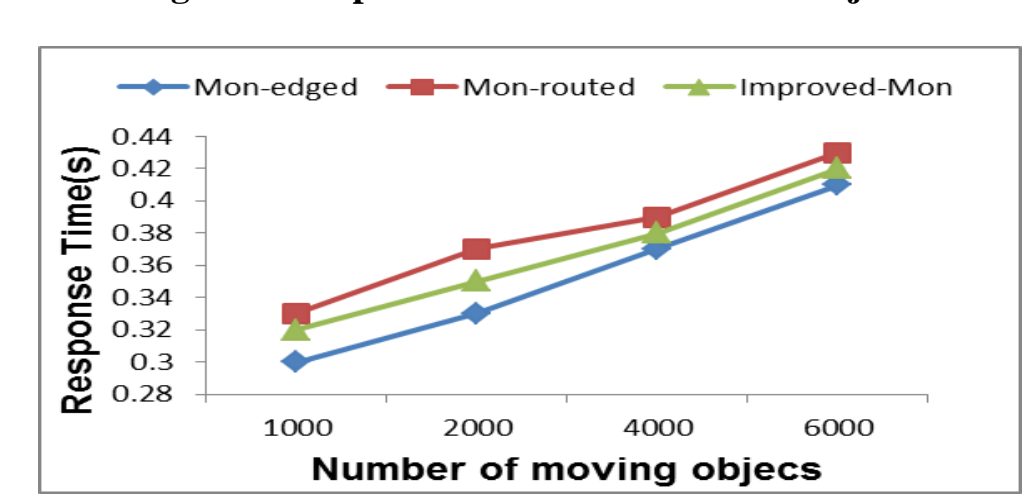

Figure 4. Response Time vs Query Window Size

For global trajectory query, we are given an object identifier, and return to its trajectory information. The relationships of response time between the numbers of moving objects shown in Figure 5.

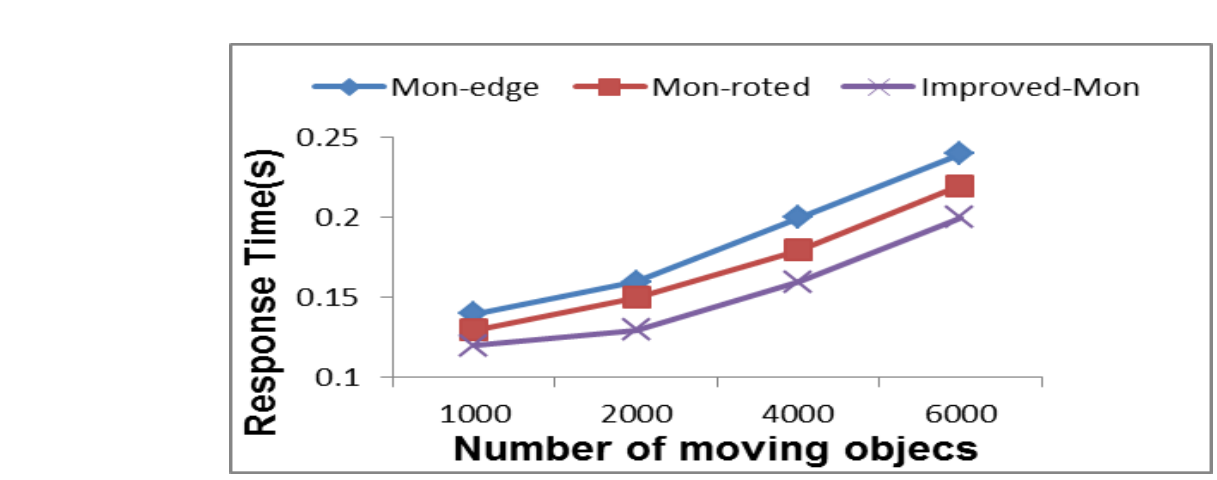

Figure 5. Response Time vs Number of Objects 
By experimental contrast Improved-MON-tree in the window query and global trajectory performance than the MON-tree has increased, mainly due to the common advantage Improved -MON-tree synthesis based on edge, and the route MON-tree the upper index structure using the edge organization, reducing the coincidence the MBR between the upper leaf node for the region query lowering the chance to traverse the entire upper quadtree, to reduce the time overhead. The underlying index structure based on the route organization, reducing the number of lower R-tree forest, the same query efficiency has improved.

Bottom increase hash + single linked list structure to reduce the time overhead for at the global trajectory query of a moving object, because based on edge or road organization for the query to a specific moving object trajectory need to traverse the entire lower R-tree forest, the Improved-MON-tree index to traverse the lower single link list, so the global trajectory of a moving object query performance has greatly improved.

\section{Conclusion}

This paper proposes an improved the indexing mechanism Improved-MON-tree on the basic of the Mon-tree.The index structure of the upper edge of the organization, the lower route-based organization, and added in the lower trajectory of moving objects. The experimental results shows that the proposed index structure has better spatial-temporal query and trajectory query performance. However, its space-overhead than MON-tree, will be the next direction of efforts.

\section{Acknowledgements}

This paper is supported by (1) The Nationat Natural Science Foundation of China No.61201315 and No.51374035, (2) Foundation of Liaoning Educational committee under the Grant No.L2010194, (3) Anshan MunicipaLSatural Science Foundation under the Grant No.2011MS21.

\section{References}

[1] C. Jidong and M. Xraofeng, "Update-fficient Indexing of Moving Objects in Road Networks", GeoInformatica, vol. 13, no. 4, (2009). pp. 397-424.

[2] L. Hu, Y. Li, J. Xiong and G.Fang, "A Filling Algorithm of Mining Constraint Frequent Neighboring Class Set", International Journat of Digital Content Technology and its Applications, vol. 6, no. 16, (2012), pp. 188-195.

[3] W. Liu, W. Li, Y. B, Z. Chen and Y. He, "Cluster-based Superseding Nearest Neighbor Queries on Uncertain Data", Journal of Convergence Information Technology, vol. 7, no. 17, (2012), pp. 392-401.

[4] D. Zhiming and L. Xiaonan, "Indexing the Historical, Current, and Future Locations of Network-Constrained Moving Objects", Journal of Software, vol. 20, no. 12, (2009), pp. 3193- 3204.

[5] L. Zhenhai and Y. Jianqiao, "Full-time Position Index Model of Moving Objects on Fixed Network", Computer Engineering, vol. 37, no. 3, (2011), pp. 67-69.

[6] T. Cho and S. Choi, "A Multi-path Hybrid Routing Algorithm in Network Routing", International Journal of Hybrid Information Technology, vol. 5, no. 3, (2012), pp. 41-46.

[7] T. Xiaotian and Y. Jian-qiao, "Efficient Index Model of Moving Object Full-time State for Road Network", Computer Engineering, vol. 38, no. 6, (2012), pp. 53-55.

[8] J. Chen and X. Meng, "Indexing Future Trajectories of Moving Objects in a Constrained Network", JCST, vol. 22, no. 2, (2007), pp. 245-251.

[9] V. Almeida and R. H. Güting, "Indexing the Trajectories of Moving Objects in Networks", SSDBM'04, Santorini island, Greece, (2004).

[10] L. Guohui and Z. Xiya, "Indexing Moving Objects Trajectories on Fixed Networks", Journal of Computer Research and Development, vol. 43, no. 5, (2006), pp. 828-833.

[11] L. Feng and L. Lei, "Spatiotemporal index for moving objects in networks: IMon-tree", Journal of Computer Applications, vol. 32, no. 8, (2012), pp. 2205-2208. 
[12] G. Jingfeng and W. Jianzhao, "Indexing Scheme of Moving Objects on Fixed Networks", Computer Science, vol. 33, no. 7, (2006), pp. 68-70.

[13] Z. Wang and Qiang Niu, "Research on Hybrid Query Expansion Algorithm", International Journal of Hybrid Information Technology, Vol. 5, No. 2, (2012),pp.207-212.

[14] Zhang Jingmin,Wang Peichong,Lu Fengjia, "Indexing method of moving objects on networks" Computer Engineering and Applications,Vol.45,No.12(2009),pp.144-146.

[15] K. S. Kim, "Fast Indexing and Updating Method for Moving Objects on Road Networks", WISE'03, Roma, Italy, (2003).

[16] R. Hartmut Güting, V. Teixeira de Almeida and Z. Ding, "Modeling and querying moving objects in networks", The International Journal on Very Large Data Bases, vol. 15, no. 2, (2006), pp. 165-190.

[17] R. H. Güting, T. BEHR and D. C. Secondo, "A platform for moving objects database research and for publishing and integrating research implementations", IEEE Data Engineering Bulletin, vol. 33, no. 2, (2010), pp. 56-63.

[18] E. Frentzos, “Indexing Objects Moving on Fixed Networks”, SSTD’03, Santorini Island, Greece, (2003).

[19] E. Akbari Baniani and A. Chalechale, "Hybrid PSO and Genetic Algorithm for Multilevel Maximum Entropy Criterion Threshold Selection", International Journal of Hybrid Information Technology, vol.. 6, no. 5, (2013), pp. 131-140.

[20] N. Kerdprasop and K. Kerdprasop, "A Robust Tree Induction Method Based on Heuristics and Cluster Analysis”, International Journal of Database Theory and Application, vol. 5, no. 2, (2012), pp. 17-34.

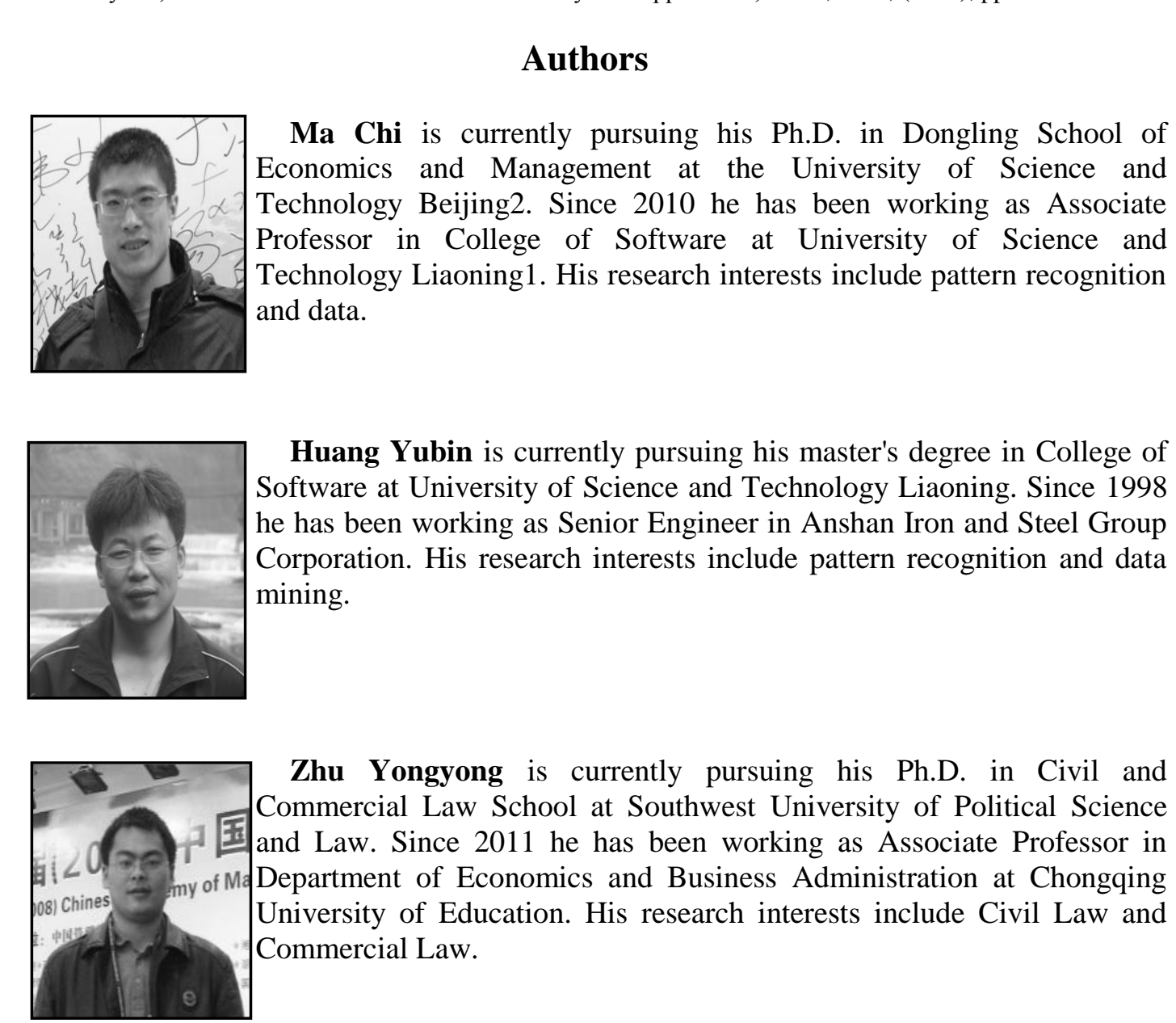


International Journal of Hybrid Information Technology

Vol.7, No.4 (2014)

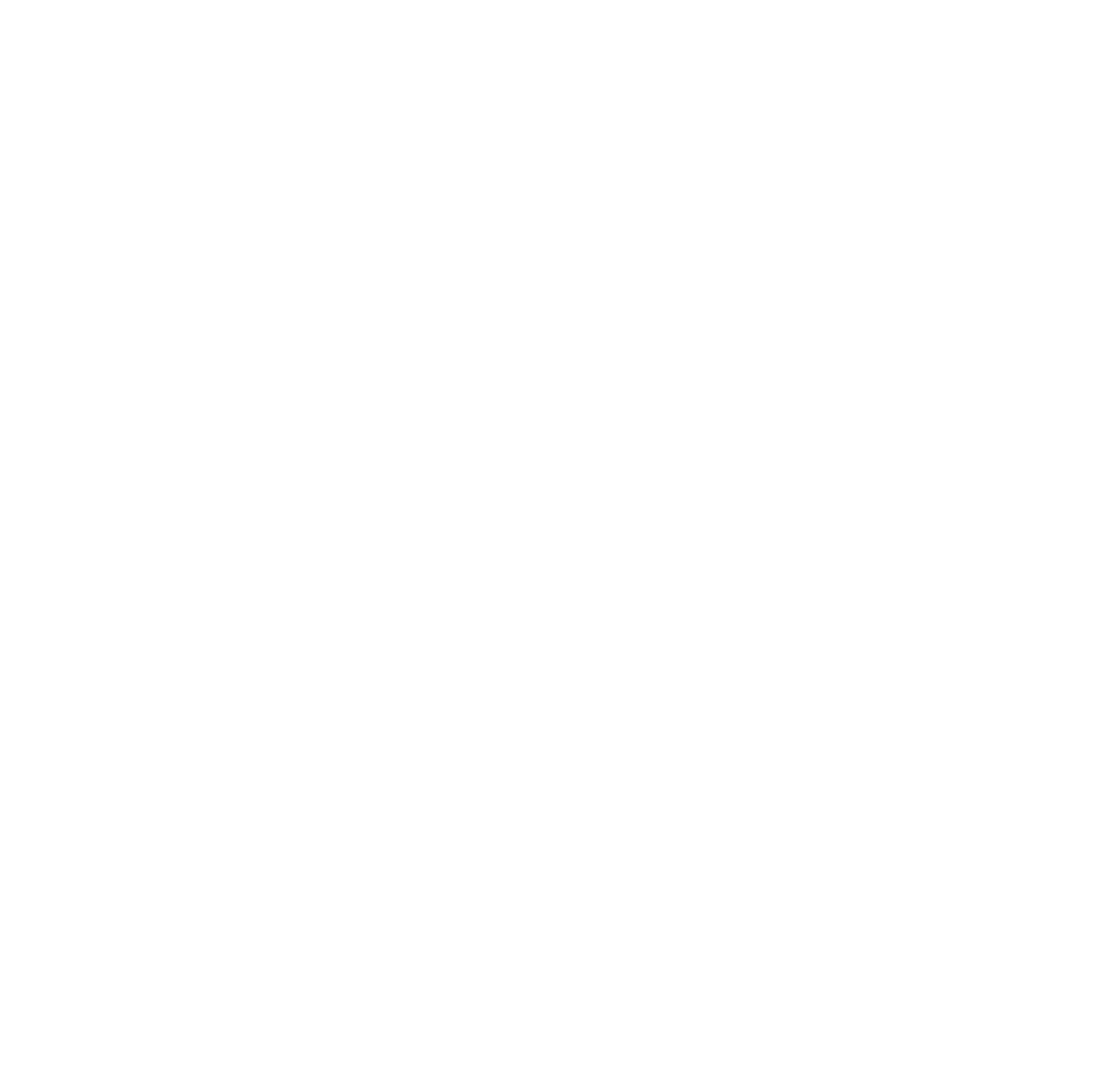

